\section{Evaluation of agreement between intraocular pressure measurements using Goldmann applanation tonometry and Goldmann correlated intraocular pressure by Reichert's ocular response analyser}

JR Ehrlich ${ }^{1}$, S Haseltine', M Shimmyo ${ }^{2}$ and NM Radcliffe ${ }^{1}$

\begin{abstract}
Purpose To compare agreement of intraocular pressure (IOP) measurements using Goldmann applanation tonometry (GAT) and Goldmann correlated intraocular pressure generated (IOPg) by the Reichert ocular response analyser (ORA).

Methods Consecutive patients presenting for glaucoma evaluation underwent ORA assessment followed by examination including GAT. For each ORA assessment, measurements were taken until a waveform score (WS) of 6.5 was obtained or until five measurements were obtained per eye. The relationship between GAT and IOPg and the influence of the WS upon this relationship was evaluated. A Bland-Altman plot and linear regression were used to determine agreement between GAT and IOPg.

Results A total of 518 eyes of 260 patients were included in the final analysis. Increasing WS was found to predict a smaller difference between GAT and IOPg $(\beta=-0.2, P \leqslant 0.001)$.

Selecting the highest WS among ORA assessments of each eye, WS continued to predict concordance between GAT and IOPg $(\beta=-0.2, P=0.006)$. The mean IOP difference between methods was $0.1 \mathrm{~mm} \mathrm{Hg}( \pm 0.3)$, which was found to be statistically insignificant $(P=0.391)$. This relationship
\end{abstract}

between GAT and IOPg was successfully validated using a second distinct data set of 100 eyes. GAT and IOPg measurements varied by $2 \mathrm{~mm} \mathrm{Hg}$ or less in $53.9 \%$ of eyes and $5 \mathrm{~mm} \mathrm{Hg}$ or less in $\mathbf{9 2 . 3 \%}$ of eyes.

Conclusion In clinical practice IOPg is strongly related to GAT. Although higher WS is indicative of greater IOPg/GAT concordance, its influence is minimal. This study does not support the use of a specific WS cutoff to determine quality of an IOPg measurement. Eye (2010) 24, 1555-1560; doi:10.1038/eye.2010.83; published online 28 May 2010

Keywords: intraocular pressure; ocular tonometry; ocular response analyser; Goldmann

\section{Introduction}

Intraocular pressure (IOP) is currently the only modifiable risk factor for glaucoma, a disease that is the second leading cause of blindness worldwide. ${ }^{1,2}$ As IOP reduction is the mainstay of treatment, accurate IOP assessment is important in monitoring the efficacy of therapy and for assessing the risk of glaucomatous progression..$^{3-6}$ Goldmann applanation tonometry (GAT) is currently the clinical gold standard for IOP assessment. ${ }^{7}$ Despite its widespread use, GAT has several limitations,
'Department of Ophthalmology, Weill Cornell Medical College, New York, NY, USA

${ }^{2}$ Department of Ophthalmology, New York Medical College, Valhalla, NY, USA

Correspondence: N Radcliffe, Department of Ophthalmology, Weill Cornell Medical College, 1305 York Avenue, New York, NY 10021, USA

Tel: + 646962 2020; Fax: +6469620603. E-mail: nmr9003@ med.cornell.edu

Received: 15 October 2009 Accepted in revised form: 30 April 2010 Published online: 28 May 2010 
including confounding related to corneal thickness, calibration errors, and concerns related to contamination and sanitation. ${ }^{8-11}$

The non-contact ocular response analyser (ORA: Reichert Ophthalmic Instruments, Buffalo, NY, USA) provides a corneal compensated IOP measurement (IOPcc) that avoids several of the imitations of GAT. $^{7,8,10,12-15}$ The ORA has received attention in the areas of glaucoma, corneal disorders, and refractive surgery. It also provides a Goldmann correlated IOP (IOPg) value and a waveform score (WS) designed to guide the clinician in selecting high quality measurements. Although the determination of a more accurate IOP assessment is appealing to eye care professionals, the thought of abandoning GAT is concerning due to issues of continuity of care. As clinicians looking to move towards corneal compensated IOP measurement in glaucoma management will likely desire a period of transition, it is important to first determine how well IOPg approximates GAT. This study sought to evaluate the agreement of GAT and IOPg in a population of patients under clinical evaluation for glaucoma. Furthermore, this study sought to determine the value of the WS and other variables in determining which measurements best approximate GAT.

\section{Methods}

Approval for this study was obtained from the Institutional Review Board at Weill Cornell Medical College and New York Presbyterian Hospital. A total of 518 eyes of 260 consecutive patients over 18 years of age undergoing corneal hysteresis $(\mathrm{CH})$ measurement as part of glaucoma evaluation from June 2009 to August 2009 at Weill Cornell Medical College were selected for inclusion in this retrospective cross-sectional study. Using the same criteria, 100 eyes of 52 patients were included in a separate second data set that was used to confirm results. Primary clinical diagnoses were obtained from retrospective chart review.

All subjects underwent ORA measurement directly followed by ophthalmic examination including GAT. For each ORA assessment, measurements were obtained until a WS of 6.5 was obtained or until five measurements were obtained per eye. As no published recommendations exist for choosing measurements based on WS, 6.5 was arbitrarily chosen as a cutoff score in this study based on office protocol. Topical anaesthetic and fluorescein was placed in each eye before GAT, which was performed immediately after ORA evaluation per office protocol. The GAT used for the study was calibrated monthly for errors of $\pm 0.5 \mathrm{~mm} \mathrm{Hg}$ using the manufacturer's standard check weight and protocol. ${ }^{16}$ GAT measurements were performed by one of three ophthalmologists. As the ORA assessment was performed primarily to measure $\mathrm{CH}$ (which was the only ORA-generated value documented in the patient record), the examiner measuring GAT was masked to IOPg during IOP assessment.

Charts for all study patients were reviewed for demographic and ocular data recorded on the day of ORA assessment. The most recent central corneal thickness (CCT) measurements obtained by ultrasound pachymetry (DGH Technology, Exton, PA, USA) and 24-2 SITA-standard automatic perimetry with the Humphrey Field Analyser II (Carl Zeiss Meditec, Dublin, CA, USA) results were obtained by chart review. The visual field index mean deviation was recorded from the most recent field test for each eye.

Statistical analyses were performed using SPSS statistical package (SPSS Inc., Chicago, IL, USA) and Stata/11 IC (StataCorp LP, College Station, TX, USA). Paired $t$-tests and simple linear regression analyses were performed. Multiple linear regression was used to validate the modelled relationship between GAT and IOPg on a second data set. To test the null hypothesis that the regression lines from the two data sets were equal, we built a multiple linear model including an indicator variable corresponding to each patient's data set of origin and allowing for interaction between data set of origin and mean IOP in predicting the difference between GAT and IOPg. The indicator variable tests equality of intercepts and the interaction term tests equality of slopes between the two lines. ${ }^{17}$ A Bland-Altman plot was constructed to determine agreement between GAT and IOPg assessments. Linear regression was used to calculate regression-based limits of agreement. All statistical tests were two-sided, with a 0.05 level for statistical significance.

\section{Results}

Patient characteristics, including primary glaucoma diagnoses, are presented in Table 1 . To determine whether WS can be used to predict concordance of IOPg and GAT measurements, we performed simple linear regression using all ORA assessments conducted during the study period. Patients underwent ORA measurement 2.3 times per eye on average. Increasing WS was found to predict a smaller absolute value difference between GAT and IOPg $(\beta=-0.2,95 \% \mathrm{CI}=-0.2,-0.1, P \leqslant 0.001)$. The highest WS was then selected from among all assessments of each eye. Simple linear regression was again used to model the relationship between highest WS and absolute value difference between GAT and IOPg. When eyes were selected based on highest WS, WS continued to predict concordance between GAT and IOPg $(\beta=-0.2,95 \% \mathrm{CI}-0.3,0.0, P=0.006)$. The mean 
Table 1 Patient characteristics

\begin{tabular}{lccc}
\hline & Mean & SD & Range \\
\hline Age (years) & 63.4 & 14.1 & $23-93$ \\
Sex (\% male) & 35.7 & & \\
CCT ( $\mu \mathrm{m})$ & 542.6 & 41.6 & $419-687$ \\
CH & 9.16 & 1.8 & $2.8-14.7$ \\
Mean deviation $(\mathrm{db})$ & -5.3 & 7.1 & -32.6 to 18.9 \\
GAT (mm Hg) & 15.6 & 4.3 & $6-44$ \\
IOPg (mm Hg) & 15.5 & 5.4 & $4.1-58.9$ \\
& & & \\
Primary glaucoma diagnosis & & & \\
$\quad$ Open-angle glaucoma & $46 \%$ & & \\
$\quad$ Glaucoma suspect & $41 \%$ & & \\
$\quad$ Ocular hypertension & $7 \%$ & & \\
$\quad$ Anatomic narrow angle & $6 \%$ & & \\
\hline
\end{tabular}

WS for all ORA assessments was $5.7( \pm 2.5$ (SD)), whereas the mean WS was $7.5( \pm 1.6(\mathrm{SD}))$ when the highest score was selected for each eye. On the basis of the regression equation when all ORA assessments were considered, an increase in WS from 0 (lowest possible score) to 10 (highest possible score) predicts a decrease in variance between GAT and IOPg of only $1.6 \mathrm{~mm} \mathrm{Hg}$.

As WS may be a good predictor of ORA assessment accuracy, we conducted analyses of GAT-IOPg concordance using data from the ORA evaluation with the best WS for each eye examined. The 518 eyes examined had a mean IOP of $15.6 \mathrm{~mm} \mathrm{Hg}( \pm 4.3$ (SD)) by GAT and $15.5 \mathrm{~mm} \mathrm{Hg}( \pm 5.4(\mathrm{SD}))$ by IOPg. The mean IOP difference between the two methods was $0.1 \mathrm{~mm} \mathrm{Hg}$, which was found to be a statistically insignificant difference by paired $t$-test analysis $(P=0.391)$. Figure 1 uses cutoff values to illustrate the percentage of eyes within defined limits of variation between GAT and IOPg. GAT and IOPg measurements varied by $2 \mathrm{~mm} \mathrm{Hg}$ or less in $53.9 \%$ of eyes and by a maximum of $5 \mathrm{~mm} \mathrm{Hg}$ in $92.3 \%$ of eyes.

As variation between GAT and IOPg differed significantly as a function of IOP $(P<0.001)$, regressionbased limits of agreement were calculated. The mean difference between GAT and IOPg values was $0.1 \mathrm{~mm} \mathrm{Hg}$ with $95 \%$ limits of agreement from -0.2 to 0.4 . To determine if differences between GAT and IOPg values were also related to levels of $\mathrm{CCT}, \mathrm{CH}$ and/or age, the difference between GAT and IOPg was regressed on each of these independent variables. Although mean IOP $(P<0.001)$ and CCT $(P<0.001)$ were significantly associated with the difference between GAT and IOPg measurements, $\mathrm{CH}(P=0.596)$ and age $(P=0.874)$ were not (Table 2). A Bland-Altman plot showed moderate variation between GAT and IOPg (Figure 2).

IOPg was shown to overestimate GAT at pressures above $16 \mathrm{~mm} \mathrm{Hg}$ and underestimate GAT at pressures below this cutoff (regression equation:

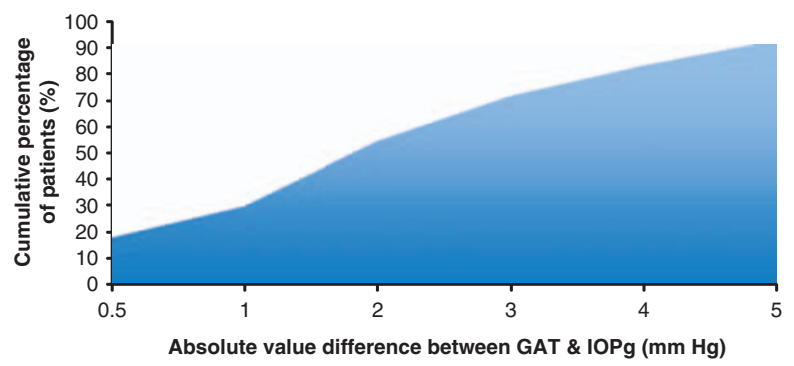

Figure 1 Percent of eyes in which GAT and IOPg measurements vary by given pressure. GAT and IOPg measurements vary by $2 \mathrm{~mm} \mathrm{Hg}$ or less in $53.9 \%$ of eyes and by $5 \mathrm{~mm} \mathrm{Hg}$ or less in $92.3 \%$ of eyes.

Table 2 Simple linear models to predict GAT-IOPg difference

\begin{tabular}{lccr}
\hline Variable & \multicolumn{3}{c}{ GAT-IOPg difference } \\
\cline { 2 - 4 } & Model $\beta$ & $95 \%$ CI & P-value \\
\hline Mean IOP & -0.25 & $-0.30,-0.19$ & $<0.001$ \\
CCT & -0.02 & $-0.02,-0.01$ & $<0.001$ \\
CH & 0.04 & $-0.11,0.19$ & 0.596 \\
Age & 0.00 & $-0.02,0.02$ & 0.874 \\
\hline
\end{tabular}

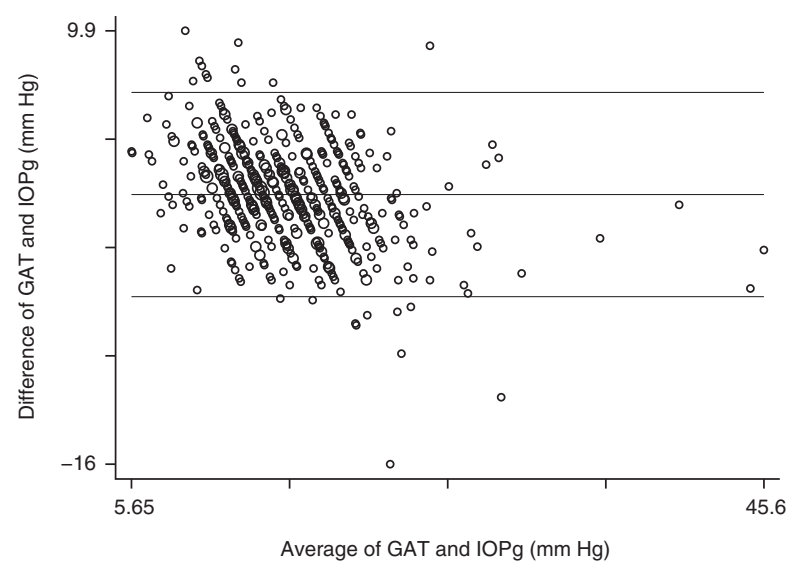

Figure 2 Bland-Altman plot of GAT and IOPg. The BlandAltman plot assesses agreement of GAT and IOPg for measurement of IOP. Mean difference $=0.1(95 \% \mathrm{CI}-0.2,0.4)$; reference range for difference -6.0 to 6.2 . Pitman's test $P$-value $\leqslant 0.001$.

$(\mathrm{GAT}-\mathrm{IOPg})=3.941-0.246 \times($ mean IOP measurement $)$, $P \leqslant 0.001$ ). Table 3 illustrates the expected bias in IOP measurement at several levels of IOP. Of note, the mean IOP in this study was close to $15 \mathrm{~mm} \mathrm{Hg}$ and at this pressure there is an estimated bias of approximately $0.3 \mathrm{~mm} \mathrm{Hg}( \pm 0.25$ (95\% limits of agreement)) between methods.

Using a second data set of 100 eyes, we sought to validate the relationship modelled between GAT and IOPg in this study. Simple linear regression using the second data set resulted in the following equation: $(\mathrm{GAT}-\mathrm{IOPg})=3.667-0.242 \times($ mean IOP measurement $)$. 
Table 3 Estimates for differences between GAT and IOPg at several IOP levels

\begin{tabular}{lc}
\hline$I O P(\mathrm{~mm} \mathrm{Hg})$ & Estimated difference $(95 \% \mathrm{CI})$ \\
\hline 10 & $1.5(1.1,1.9)$ \\
15 & $0.3(0.0,0.5)$ \\
20 & $-1.0(-1.3,-0.6)$ \\
25 & $-2.2(-2.8,-1.6)$ \\
\hline
\end{tabular}

Multiple linear regression was used to test for coincidence of the regression lines from the two data sets. There was no statistically significant association of the data set indicator variable (equality of line intercepts; $P=0.524)$ or the data set mean IOP interaction term (equality of line slopes; $P=0.659$ ) with the difference between GAT and IOPg measurements; this indicates that the regression lines of the two data sets were not significantly different.

\section{Discussion}

In this clinical study of IOP measurement in a population of patients under evaluation for glaucoma, IOP assessments with GAT and IOPg values showed good agreement on most measures and were not significantly different upon paired analysis. The difference between GAT and IOPg values, however, was significantly associated with mean IOP. Thus, the magnitude and direction of bias between GAT and IOPg values was found to vary as a function of IOP. We successfully validated the modelled relationship between GAT and IOPg using a second distinct data set.

The WS was a significant predictor of agreement between the two measures; however, the magnitude of the predicted decrease in variance between GAT and IOPg using a higher WS was small. Although this result supports the use of WS in selecting the most accurate ORA measurement during a given measurement session, it does not provide a specific cutoff value for ORA measurement quality, as IOPg and GAT were closely related across the entire spectrum of WS scores.

In order for two measures to show a strong agreement, they need to be reliable. Some past research suggests that $\mathrm{IOPg}$ is a reliable measurement of IOP and its repeatability coefficient is comparable with that of GAT. ${ }^{18}$ The intrasession repeatability of GAT and IOPg measurements have generally been found to be within about $2 \mathrm{~mm} \mathrm{Hg} .{ }^{18-24}$ In addition, inter-examiner variability or bias is an issue that is theoretically eliminated with the use of an objective assessment of IOP such as ORA-generated IOPg that would eliminate the need for masked IOP assessment protocols in the research setting. In a population-based epidemiologic study, Dielemans et $a l^{19}$ investigated GAT test-retest reliability and found a mean intra-observer variation of $1.64 \mathrm{~mm} \mathrm{Hg}$ and inter-observer variation of $1.79 \mathrm{~mm} \mathrm{Hg}$. Thorburn $^{22}$ investigated inter-examiner variability of GAT measurements by two experienced ophthalmologists and found a difference of $2 \mathrm{~mm} \mathrm{Hg}$ or more in $40 \%$ of eyes and $3 \mathrm{~mm} \mathrm{Hg}$ or more in $17 \%$ of eyes examined. Similarly, Phelps and Phelps ${ }^{21}$ reported a difference between the GAT measurements of two examiners of at least $2 \mathrm{~mm} \mathrm{Hg}$ in $50 \%$ of eyes and $3 \mathrm{~mm} \mathrm{Hg}$ in $30 \%$ of eyes. Accordingly, the measurement variability between IOPg and GAT in this study is similar to previously reported inter-examiner variability of GAT measurements.

Tonnu $e a^{25}$ studied the reproducibility of several IOP measurement techniques by comparing GAT with Tono-Pen tonometry, ocular blood flow tonometry, non-contact tonometry (NCT) and a second Goldmann observer. The authors presented estimates of IOP differences between tonometry methods at various levels of IOP, as in the current investigation. Of note, at $15 \mathrm{~mm} \mathrm{Hg}$ - approximately the mean IOP in the current study - estimated GAT-IOPg agreement in this study was superior to agreement between GAT and all other IOP measurement techniques considered by Tonnu et al. Moreover, this study determined a mean difference of $0.1 \mathrm{~mm} \mathrm{Hg}$ between GAT and IOPg values, compared with $0.7 \mathrm{~mm} \mathrm{Hg}$ between GAT and NCT in the study by Tonnu et al..$^{25}$ In addition, in two separate studies, Jorge et $\mathrm{al}^{27}$ demonstrated a difference of $0.1 \mathrm{~mm} \mathrm{Hg}$ between GAT and NCT values among normal ${ }^{26}$ and glaucomatous subjects using the same NCT machine. However, using a different NCT machine (from the same manufacturer) to study the same cohort of normal subjects, the investigators reported a mean GAT-NCT difference of $0.8 \mathrm{~mm} \mathrm{Hg} .^{26}$ Thus, it is clear that IOP measurement agreement will depend greatly on the protocol and equipment used.

A growing literature regarding $\mathrm{CH}$ measurement has demonstrated a potentially expanding role for the ORA in ophthalmic management. $\mathrm{CH}$ is lower in glaucoma damage, acquired optic nerve pitting, and progressive glaucoma. ${ }^{2,15,28-31}$ In glaucoma patients with low hysteresis, there is greater backward bowing of the lamina cribrosa in response to transient IOP elevation. ${ }^{32}$ Furthermore, $\mathrm{CH}$ measurements may be useful in identifying patients with keratoconus and post-LASIK ectasia, as well as for monitoring biomechanical corneal properties before and after keratorefractive procedures. ${ }^{14,33-36}$ By compensating for $\mathrm{CH}$ and other properties, the ORA produces a corneal compensated IOP. Although $\mathrm{CH}$ is moderately correlated with CCT, $\mathrm{CH}$ and corneal compensated IOP measurements are less affected by corneal oedema than are GAT measurements. ${ }^{33,37,38}$ Furthermore, IOPcc may be one of the most appropriate measures of IOP before and after keratorefractive procedures and in eyes with keratoconus or corneal oedema. ${ }^{14,33-36,39}$ In light of these and other 
findings, the ORA is an appealing tool for the measurement of IOP and ocular biomechanical characteristics.

It is important to note that the observed bias between GAT and IOPg could affect clinical management. This may be particularly significant for patients with borderline IOP, as IOPg values tend to exceed GAT at higher pressures in this study. Nevertheless, the bias detected between GAT and IOPg is similar to some previously reported inter- and intra-examiner GAT biases. ${ }^{19,21,22}$ Accordingly, the variation between IOPg and GAT may be acceptable to clinicians who are accustomed to this sort of variability. ${ }^{40}$ Future work should examine the test-retest repeatability of IOPg to determine if it provides a more repeatable measurement of IOP.

Given the likely redundancy of measuring both IOPg and GAT, the exclusive use of the ORA might offer several advantages to the clinician. In addition to measuring hysteresis and providing several objective measures of IOP, the ORA does not require the use of fluorescein and topical anaesthetic, nor does the instrument contact the patient's cornea or require sterilization (although a forehead guide is used). However, as physicians consider adapting to new technologies several issues arise. Clinical gold standards, such as GAT, have considerable value in the context of clinical history and continuity of care even with known inaccuracies. Furthermore, the majority of clinical research data still uses GAT measurements. By providing both IOPg and IOPcc measurements, the ORA offers clinicians a way to transition to the ORA only while still maintaining an estimate of GAT measurements through IOPg. As the inter-test variability of IOPg-GAT measurements is similar to test-retest variability of GAT, use of IOPg could help to maintain continuity of care during the adoption of this new technology without entirely abandoning the Goldmann standard.

\section{Summary}

What was known before

- There is intra- and inter-examiner variability between GAT measurements.

- GAT raises concerns about hygiene, spread of infection, and patient comfort.

- The ocular response analyser (ORA) generates a Goldmann correlated IOP (IOPg) measurement, which is a hygienic and reproducible method for measuring IOP.

\section{What this study adds}

- GAT and IOPg generally show good agreement, suggesting IOPg may be substituted for GAT.

- A higher waveform score indicates greater concordance between GAT and IOPg.

- This study does not support a specific waveform score cutoff to determine the quality of an IOPg measurement.

\section{Conflict of interest}

The authors declare no conflict of interest.

\section{References}

1 Herndon LW. Measuring intraocular pressure-adjustments for corneal thickness and new technologies. Curr Opin Ophthalmol 2006; 17(2): 115-119.

2 Congdon NG, Broman AT, Bandeen-Roche K, Grover D, Quigley HA. Central corneal thickness and corneal hysteresis associated with glaucoma damage. Am J Ophthalmol 2006; 141(5): 868-875.

3 Kass MA, Heuer DK, Higginbotham EJ, Johnson CA, Keltner JL, Miller JP et al. The Ocular Hypertension Treatment Study: a randomized trial determines that topical ocular hypotensive medication delays or prevents the onset of primary open-angle glaucoma. Arch Ophthalmol 2002; 120(6): 701-713; discussion 829-830.

4 Leske MC, Heijl A, Hussein M, Bengtsson B, Hyman L, Komaroff E. Factors for glaucoma progression and the effect of treatment: the early manifest glaucoma trial. Arch Ophthalmol 2003; 121(1): 48-56.

5 The Advanced Glaucoma Intervention Study (AGIS) 7. The relationship between control of intraocular pressure and visual field deterioration. The AGIS Investigators. Am J Ophthalmol 2000; 130(4): 429-440.

6 Collaborative Normal-Tension Glaucoma Study Group. Comparison of glaucomatous progression between untreated patients with normal-tension glaucoma and patients with therapeutically reduced intraocular pressures. Am J Ophthalmol 1998; 126(4): 487-497.

7 Doughty MJ, Zaman ML. Human corneal thickness and its impact on intraocular pressure measures: a review and meta-analysis approach. Surv Ophthalmol 2000; 44(5): 367-408.

8 Medeiros FA, Weinreb RN. Evaluation of the influence of corneal biomechanical properties on intraocular pressure measurements using the ocular response analyzer. J Glaucoma 2006; 15(5): 364-370.

9 Martinez-de-la-Casa JM, Garcia-Feijoo J, Fernandez-Vidal A, Mendez-Hernandez C, Garcia-Sanchez J. Ocular response analyzer versus Goldmann applanation tonometry for intraocular pressure measurements. Invest Ophthalmol Vis Sci 2006; 47(10): 4410-4414.

10 Kotecha A, Elsheikh A, Roberts CR, Zhu H, Garway-Heath DF. Corneal thickness- and age-related biomechanical properties of the cornea measured with the ocular response analyzer. Invest Ophthalmol Vis Sci 2006; 47(12): 5337-5347.

11 Broman AT, Congdon NG, Bandeen-Roche K, Quigley HA. Influence of corneal structure, corneal responsiveness, and other ocular parameters on tonometric measurement of intraocular pressure. J Glaucoma 2007; 16(7): 581-588.

12 Moreno-Montañés J, Maldonado MJ, García N, Mendiluce L, García-Gómez PJ, Seguí-Gómez M. Reproducibility and clinical relevance of the ocular response analyzer in nonoperated eyes: corneal biomechanical and tonometric implications. Invest Ophthalmol Vis Sci 2008; 49(3): 968-974.

13 Streho M, Dariel R, Giraud J, Verret C, Fenolland J, Crochelet $\mathrm{O}$ et al. Evaluation of the Ocular Response Analyzer in ocular hypertension, glaucoma, and normal populations. Prospective study on 329 eyes. J Fr Ophtalmol 2008; 31(10): 953-960. 
14 Pepose JS, Feigenbaum SK, Qazi MA, Sanderson JP, Roberts CJ. Changes in corneal biomechanics and intraocular pressure following LASIK using static, dynamic, and noncontact tonometry. Am J Ophthalmol 2007; 143(1): 39-47.

15 Luce DA. Determining in vivo biomechanical properties of the cornea with an ocular response analyzer. J Cataract Refract Surg 2005; 31(1): 156-162.

16 Haag-Streit. Goldmann Applantation Tonometer Instruction Manual 2003. Available at:www.haag-streit.com. (Accessed 18 January 2010).

17 Kleinbaum DG, Kupper LL, Nizam A, Muller KE. Applied Regression Analysis and Multivariable Methods, 4th ed. Duxbury Press, Belmont, CA, USA, 2007.

18 Kynigopoulos M, Schlote T, Kotecha A, Tzamalis A, Pajic B, Haefliger I. Repeatability of intraocular pressure and corneal biomechanical properties measurements by the ocular response analyser. Klin Monatsbl Augenheilkd 2008; 225(5): 357-360.

19 Dielemans I, Vingerling JR, Hofman A, Grobbee DE, de Jong PT. Reliability of intraocular pressure measurement with the Goldmann applanation tonometer in epidemiological studies. Graefes Arch Clin Exp Ophthalmol 1994; 232(3): 141-144.

20 Moses RA, Liu CH. Repeated applanation tonometry. Am J Ophthalmol 1968; 66(1): 89-91.

21 Phelps CD, Phelps GK. Measurement of intraocular pressure: a study of its reproducibility. Albrecht Von Graefes Arch Klin Exp Ophthalmol 1976; 198(1): 39-43.

22 Thorburn W. The accuracy of clinical applanation tonometry. Acta Ophthalmol (Copenh) 1978; 56(1): 1-5.

23 Berry V, Drance SM, Wiggins RL, Schulzer M. A study of the errors of applanation tonometry and tonography on two groups of normal people. Can J Ophthalmol 1966; 1(3): 213-220.

24 Whitacre MM, Stein R. Sources of error with use of Goldmann -type tonometers. Surv Ophthalmol 1993; 38(1): 1-30.

25 Tonnu P, Ho T, Sharma K, White E, Bunce C, Garway-Heath D. A comparison of four methods of tonometry: method agreement and interobserver variability. $\mathrm{Br} J$ Ophthalmol 2005; 89(7): 847-850.

26 Jorge J, Díaz-Rey JA, González-Méijome JM, Almeida JB, Parafita MA. Clinical performance of the Reichert AT550: a new non-contact tonometer. Ophthalmic Physiol Opt 2002; 22(6): 560-564.

27 Jorge J, González-Méijome JM, Díaz-Rey JA, Almeida JB, Ribeiro P, Parafita MA. Clinical performance of non-contact tonometry by Reichert AT550 in glaucomatous patients. Ophthalmic Physiol Opt 2003; 23(6): 503-506.

28 Bochmann F, Ang GS, Azuara-Blanco A. Lower corneal hysteresis in glaucoma patients with acquired pit of the optic nerve (APON). Graefes Arch Clin Exp Ophthalmol 2008; 246(5): 735-738.

29 Sullivan-Mee M, Billingsley SC, Patel AD, Halverson KD, Alldredge BR, Qualls C. Ocular Response Analyzer in subjects with and without glaucoma. Optom Vis Sci 2008; 85(6): 463-470.

30 Ang GS, Bochmann F, Townend J, Azuara-Blanco A. Corneal biomechanical properties in primary open angle glaucoma and normal tension glaucoma. J Glaucoma 2008; 17(4): 259-262.

31 Schroeder B, Hager A, Kutschan A, Wiegand W. Measurement of viscoelastic corneal parameters (corneal hysteresis) in patients with primary open angle glaucoma. Ophthalmologe 2008; 105(10): 916-920.

32 Wells AP, Garway-Heath DF, Poostchi A, Wong T, Chan KCY, Sachdev N. Corneal hysteresis but not corneal thickness correlates with optic nerve surface compliance in glaucoma patients. Invest Ophthalmol Vis Sci 2008; 49(8): 3262-3268.

33 Shah S, Laiquzzaman M, Bhojwani R, Mantry S, Cunliffe I. Assessment of the biomechanical properties of the cornea with the ocular response analyzer in normal and keratoconic eyes. Invest Ophthalmol Vis Sci 2007; 48(7): 3026-3031.

34 Kirwan C, O'Malley D, O'Keefe M. Corneal hysteresis and corneal resistance factor in keratoectasia: findings using the Reichert ocular response analyzer. Ophthalmologica 2008; 222(5): 334-337.

35 Kirwan C, O'Keefe M. Corneal hysteresis using the Reichert ocular response analyser: findings pre- and post-LASIK and LASEK. Acta Ophthalmol 2008; 86(2): 215-218.

36 Kirwan C, O'Keefe M. Measurement of intraocular pressure in LASIK and LASEK patients using the Reichert Ocular Response Analyzer and Goldmann applanation tonometry. J Refract Surg 2008; 24(4): 366-370.

37 Touboul D, Roberts C, Kérautret J, Garra C, Maurice-Tison $\mathrm{S}$, Saubusse E et al. Correlations between corneal hysteresis, intraocular pressure, and corneal central pachymetry. J Cataract Refract Surg 2008; 34(4): 616-622.

38 Lu F, Xu S, Qu J, Shen M, Wang X, Fang H et al. Central corneal thickness and corneal hysteresis during corneal swelling induced by contact lens wear with eye closure. Am J Ophthalmol 2007; 143(4): 616-622.

39 Mollan SP, Wolffsohn JS, Nessim M, Laiquzzaman M, Sivakumar S, Hartley S et al. Accuracy of Goldmann, ocular response analyser, Pascal and TonoPen XL tonometry in keratoconic and normal eyes. Br J Ophthalmol 2008; 92(12): 1661-1665.

40 Brandt JD. The myth of clinical precision. Ophthalmology 2009; 116(1): 1-2.e1. 